\title{
Além da auditoria financeira: fatores que podem influenciar a presença das grandes firmas de auditoria contábil no mercado de asseguração externa dos relatórios de sustentabilidade no Brasil
}

\author{
Gabriela Borges Silveira (D), Hans Michael Van Bellen (D), Alex Mussoi Ribeiro \\ Universidade Federal de Santa Catarina, Florianópolis-SC, Brasil.
}

\section{$\bigotimes$}

'gabi_contiante@yahoo.com.br

2hans.michael@ufsc.br

3alex.mussoi@ufsc.br

Editado por:

Orleans Silva Martins

Paulo Roberto da Cunha

\section{Resumo}

Objetivo: Esta pesquisa busca analisar os fatores que podem influenciar a presença das grandes firmas de auditoria contábil no mercado de asseguração externa dos Relatórios de Sustentabilidade (RS) no Brasil.

Método: A amostra compõe-se de 47 companhias de capital aberto que asseguraram seus RS no período de 2012 a 2018. Os dados foram analisados por meio das técnicas estatísticas Anacor, HOMALS e regressão hierárquico logístico para dados em painel. Resultados: Os resultados apontaram que a prestação conjunta de auditoria contábil e asseguração externa dos RS, bem como o isomorfismo mimético são fatores que afetam positivamente a presença das firmas Big-4 no mercado de asseguração externa dos RS no Brasil, caracterizando a vantagem competitiva dos provedores de contabilidade sobre os demais provedores nesse emergente mercado.

Contribuições: Este trabalho traz importantes implicações para profissionais e reguladores da área contábil, pois o reconhecimento das firmas de auditoria contábil no mercado de asseguração externa dos RS deve promover o interesse em desenvolver apoio institucional a essa prática.

Palavras-chave: Asseguração externa dos relatórios de sustentabilidade. Provedores de asseguração. Capital reputacional das firmas Big-4.

\section{Como citar:}

Silveira, G. B. ., Van Bellen, H. M. ., \& Mussoi Ribeiro, A. (2021). ALÉM DA AUDITORIA FINANCEIRA: FATORES QUE PODEM INFLUENCIAR A PRESENÇA DAS GRANDES FIRMAS DE AUDITORIA CONTÁBIL NO MERCADO DE ASSEGURAÇÃO EXTERNA DOS RELATÓRIOS DE SUSTENTABILIDADE NO BRASIL. Advances in Scientific and Applied Accounting, 14(3). Recuperado de https://asaa.anpcont.org.br/index. $\mathrm{php/asaa/article/view/831}$ 


\section{Introdução}

O número de empresas que relatam informações relacionadas ao desempenho ambiental e social aumentou nos últimos anos. A divulgação de relatórios de sustentabilidade (RS) pelas 250 maiores empresas do mundo cresceu de 35\% em 1999 para 93\% em 2017 (KPMG, 2017). Entretanto, a falta de confiança do público (Dando \& Swift, 2003) e a ausência de consistência e integridade dessas informações (Adams \& Evans, 2004) desencadeou a exigência crescente de informações mais confiáveis. Os RS, quando não fornecem medidas de desempenho fundamentadas em contribuições reais para a sustentabilidade, podem ser caracterizados como ferramentas de "greenwashing" (Papoutsi \& Sodhi, 2020).

Para reforçar a confiança e mitigar o ceticismo das partes interessadas nas informações de sustentabilidade, as empresas começaram voluntariamente a assegurar de forma externa seus RS (Simnett et al., 2009). De acordo com O'Dwyer (2011), a asseguração externa dos RS objetiva examinar, avaliar e relatar a confiabilidade e a integridade das informações acerca do desempenho de sustentabilidade de uma organização. Assim, quando é realizada de forma independente aumenta a percepção do público sobre a credibilidade dos RS (Hodge, Subramaniam \& Stewart, 2009; Simnett et al., 2009).

A asseguração externa dos RS tornou-se um mercado promissor e uma área em expansão da prática profissional para diferentes provedores, principalmente, firmas de contabilidade, certificação e consultoria especializada em sustentabilidade (Fernandez-Feijoo et al., 2016). Nesse ponto, provedores de natureza contábil demonstram ser altamente adaptáveis e capazes de inovar em mercados dinâmicos e competitivos (Andon et al., 2015). As quatro grandes firmas de contabilidade - comumente denominadas de Big-4 (PWC, KPMG, Ernest \& Young e Deloitte) - operam em nível global. Portanto, devido à sua dimensão, podem atuar em economias de escala e investir em pesquisa e desenvolvimento nas distintas formas de auditoria (Farooq \& de Villiers, 2017).

Apesar da concorrência de diferentes profissionais, diversos estudos documentaram que as grandes firmas de contabilidade se tornaram concorrentes dominantes no mercado de asseguração dos RS (Simnett et al., 2009; Fernandez-Feijoo et al., 2016; Hummel et al., 2017; Martínez-Ferrero \& García-Sánchez, 2018). O domínio da profissão contábil nesse mercado pode estar relacionado a importantes atributos profissionais, como a competência técnica para entender adequadamente a especificidade da natureza das informações de sustentabilidade divulgadas pelas empresas (Accountability, 2005; Park \& Brorson, 2005; Cohen \& Simnett, 2015) e a capacidade de agir de forma independente (Green et al., 2017).

Ressalta-se que a maioria das pesquisas sobre asseguração externa dos RS se concentraram nas diferenças entre os provedores de natureza contábil e não contábil (Gillet, 2012; Jones \& Solomon, 2010; MartínezFerrero \& García-Sánchez, 2018; O’Dwyer \& Owen, 2005; Simnett et al.,2009) e são poucas as evidências sobre quais atributos favorecem a presença das grandes firmas de contabilidade nesse mercado, considerandose as diferenças na atuação de cada firma (FernandezFeijoo et al., 2016).

Mediante o exposto, o presente estudo objetiva analisar os fatores que podem influenciar a presença das grandes firmas de auditoria contábil no mercado de asseguração externa dos RS no Brasil. Especificamente, esta análise objetiva um conjunto de características relacionado ao capital reputacional das firmas Big-4 derivadas da prestação conjunta de auditoria contábil e asseguração externa dos RS, da especialização do setor e do isomorfismo mimético. Para testar as hipóteses de pesquisa, utilizou-se um conjunto de dados em painel das companhias brasileiras de capital aberto que asseguraram seus RS no período de 2012 a 2018.

Nesta pesquisa, argumenta-se que as firmas de contabilidade Big-4 podem ser "percebidas" como experts nos trabalhos de asseguração externa dos RS, devido à atributos advindos da experiência no fornecimento de serviços tradicionais de auditoria, como a aplicação de padrões e procedimentos (Simnett et al., 2009; MartínezFerrero \& García-Sánchez, 2018), o cumprimento de requisitos de conduta profissional e independência (Peters \& Romi, 2014), no entendimento aprofundado e abrangente das operações de seus clientes (Gillet, 2012) e na especialização no setor de atividade das empresas relatoras (Green et al., 2017).

Este estudo justifica-se pela necessidade de reforçar e ampliar os resultados anteriores da emergente literatura sobre o mercado de asseguração externa dos RS, fornecendo insights adicionais sobre como as grandes firmas de contabilidade expandem o campo de atuação profissional em novos domínios de práticas adjacentes ao setor de auditoria e em diferentes contextos. Com base nas evidências documentadas por Fernandez-Feijoo et al. (2016), a pesquisa procura compreender os efeitos produzidos pela reputação das firmas Big-4, como 
prática de isomorfismo mimético, os quais são percebidos pelas empresas relatoras em um contexto social específico (setor), e discutir as razões subjacentes para que essas firmas se engajem em serviços de asseguração dos RS.

A seleção do caso brasileiro é baseada em fatores estruturais (a natureza voluntária e desregulamentada da divulgação e asseguração dos RS) e políticos (perda de atribuições da pasta de meio ambiente, redução da participação da sociedade civil e a flexibilização da fiscalização ambiental) e, ainda, na crise reputacional de empresas tidas como "referências" em sustentabilidade (por exemplo, Vale, Petrobras e Odebrechet) que trazem à tona a credibilidade e a confiabilidade das iniciativas e práticas de relato referente a Responsabilidade Social Corporativa (RSC) (Zhang \& Chen, 2019). Também é importante mencionar que credibilidade e confiança são conceitos com significados e implicações que podem variar consideravelmente entre países e culturas (Fonseca, 2010), o que reforça a motivação de estudos com amostras nacionais (Perego \& Kolk, 2012). Considerando esses aspectos, o contexto brasileiro é instigante, embora até então pouco explorado (Silveira \& Alberton, 2020).

O papel social da asseguração externa dos RS se concentra na capacidade de o provedor garantir que os interesses e as expectativas das partes interessadas, principalmente externas, relacionados à RSC sejam atendidos (Manetti \& Toccafondi, 2012); por isso, a credibilidade do profissional de asseguração é um fator relevante que afeta a confiança do público e sua percepção sobre a confiabilidade das informações relatadas (Hodge et al., 2009).

Nesse sentido, considerando a ausência de regulamentação e de padrões específicos a serem adotados pelos profissionais de contabilidade (MartínezFerrero \& García-Sánchez, 2018), as evidências desse estudo podem ser úteis para que as entidades que orientam e normatizam a profissão contábil possam refletir sobre formas de desenvolvimento institucional para esse campo de atuação.

\section{Fundamentação Teórica e Hipoteses}

Nos processos de asseguração externa dos RS um profissional independente é contratado pela administração da empresa relatora para fornecer uma opinião sobre a adequação das informações relatadas às demais partes interessadas da organização (Farooq \& de Villiers, 2019). Nessa perspectiva, para as empresas, a asseguração externa dos RS é um mecanismo cujo objetivo é gerar confiança nos demais stakeholders (Jones \& Solomon, 2010). Na maioria das jurisdições, a asseguração é um processo voluntário. E o mercado está aberto a diferentes provedores que competem por uma fatia de mercado (Farooq \& de Villiers, 2019).

Neste contexto, a literatura identificou dois amplos grupos de provedores, classificados como contábeis e não contábeis (Simnett et al., 2009; Fernandez-Feijoo et al., 2016; Martínez-Ferrero \& García-Sánchez, 2018; Farooq \& de Villiers, 2019). O grupo dos provedores de natureza contábil compreende, principalmente, as quatro grandes firmas de contabilidade - PWC, Ernest \& Young, Deloitte e KPMG (Big-4) - já os de natureza não contábil representam um grupo mais diversificado, como profissionais de engenharia e especialistas em sustentabilidade.

Acerca da execução dos processos de asseguração externa dos RS, nenhuma qualificação específica é exigida para que $\circ$ profissional realize esse tipo serviço (RuizBarbadillo \& Martínez-Ferrero, 2020). Portanto, não há consenso sobre qual categoria de provedor estaria mais capacitada para 0 trabalho ou se há uma abordagem mais apropriada para esse processo (Farooq \& de Villiers, 2019).

O'Dwyer e Owen (2005) explicam que a competitividade das firmas resultou em divergências quanto à natureza, ao escopo e à abordagem da asseguração entre contadores e não contadores. Essas divergências derivam de seu conhecimento e de sua experiência em procedimentos e no processo de asseguração como um todo, na indústria, nos negócios e nas operações de seus clientes e, ainda, no objeto de asseguração (Adams \& Evans, 2004).

Apesar da concorrência de mercado, o profissional de auditoria tornou-se provedor dominante no mercado de asseguração dos RS em todo o mundo (Simnett et al., 2009; Martínez-Ferrero \& García-Sánchez, 2018) ao passo que fatores relacionados aos atributos profissionais podem afetar a decisão da empresa sobre qual provedor contratar. E essa escolha torna-se complexa, visto que inclui argumentos com base na racionalidade econômica e coletiva (DiMaggio \& Powell, 1983).

Padrões internacionais, por exemplo ISAE300 (IAASB, 2013) e AA1000AS (AccountAbility, 2005), descrevem independência e competência técnica como requisitos básicos para que os provedores de asseguração dos RS desempenhem um trabalho eficiente. A competência técnica se refere ao conjunto de habilidades e experiência 
específicas exigidas para compreender a matéria de sustentabilidade e os métodos usados para medir e relatar essas informações (Cohen \& Simnett, 2015). Nesse sentido, contratar o auditor das demonstrações contábeis para assegurar os RS pode ser benéfico para o processo de asseguração. A experiência e o conhecimento desenvolvidos em suas tarefas de auditoria - como as que estão relacionada a áreas de ambiente operacional, risco de negócios, sistemas de controle interno - podem ser transferidos internamente para outras atividades oferecidas ao mesmo cliente (Ruiz-Barbadillo \& MartínezFerrero, 2020).

A prestação conjunta de serviços de auditoria contábil e asseguração dos RS permite economia de escopo, - que inclui a redução do tempo necessário para obter conhecimento sobre a organização relatora (Park \& Brorson, 2005) e menores taxas de asseguração (Byus et al., 2013). Jones e Solomon (2010) e Gillet (2012) constataram que a asseguração dos RS é percebida como uma extensão lógica da auditoria financeira e do domínio natural dos auditores. Portanto, a contratação de um mesmo profissional para ambos os serviços seria benéfica em termos de custo e tempo, uma vez que o auditor já estaria familiarizado com o setor, os negócios e as operações de seus clientes.

Jones e Solomon (2010) explicam que os objetivos e a metodologia empregados no processo de asseguração externa dos RS são fortemente inspirados pela auditoria financeira, motivo pelo qual as firmas de auditoria contábil podem se beneficiar do uso das mesmas técnicas de verificação de informações em seus trabalhos. FernandezFeijoo et al. (2016) concluíram que há vantagem competitiva para uma firma Big-4 entrar nesse mercado quando ela também é auditora das demonstrações contábeis. Os autores documentaram evidências de que cada empresa de contabilidade Big-4 utiliza sua rede de clientes de auditoria financeira como uma porta de entrada na asseguração externa dos RS, bem como diferenças significativas na atuação de cada grande firma de contabilidade.

Considerando que as informações de sustentabilidade são um componente altamente específico associado a cada empresa, em razão do conteúdo diversificado, complexo, único e extenso de questões relacionadas a problemas sociais, éticos e ambientais da empresa (Park \& Brorson, 2005), e que a competência técnica exigida para executar um trabalho de asseguração eficiente também deve avaliar as particularidades de cada organização (Ruiz-Barbadillo \& Martínez-Ferrero, 2020), formula-se a primeira hipótese de pesquisa.

$\mathrm{H} 1$ : A presença das grandes firmas de contabilidade (Big4) no mercado de asseguração externa dos RS é afetada positivamente pela prestação conjunta de serviços de auditoria contábil e asseguração dos RS.

No contexto da auditoria financeira, uma das formas de diferenciação da qualidade da auditoria é o grau de especialização da firma (Mayhew \& Wilkins, 2003). Casterella et al. (2004) explicam que os especialistas do setor desenvolvem um conhecimento mais profundo do que os não especialistas, como resultado de sua maior experiência e entendimento dos clientes, além de práticas de compartilhamento de conhecimento e uso de programas de auditoria padronizados para esse setor. Desse modo, os referidos autores afirmam que os especialistas são mais precisos e eficazes do que os não especialistas em seus julgamentos, pois seu entendimento sobre os negócios e setores de seus clientes permitem a criação de procedimentos de auditoria personalizados.

A especialização por indústria pode representar uma vantagem competitiva (Carson et al., 2004) ao fornecer um argumento para o desenho de estratégias de inserção das firmas de contabilidade no mercado da asseguração dos RS. Nesse sentido, vale destacar que a diversidade e a complexidade das informações de sustentabilidade estão intimamente relacionadas ao setor empresarial em que as empresas operam (Sun et al., 2017). Gürtürk e Hahn (2016) observam que grande parte das práticas, dos modelos e das técnicas de sustentabilidade para avaliação das informações pode ser relevante em alguns setores, mas não em outros. Portanto, o conjunto de habilidades e competências específicos necessário para assegurar os RS varia consideravelmente entre os setores (Pflugrath et al., 2011).

A prestação eficiente de serviços de asseguração dos RS exige que os provedores adquiram um nível de especialização específico para $\circ$ setor das empresasclientes (Green et al., 2017). Capazes de investir em pesquisa e desenvolvimento nas distintas formas de auditoria (Farooq \& de Villiers, 2017), as firmas Big-4, ao concentrarem investimento em recursos e tecnologia em um setor específico, obtém conhecimento sobre os principais aspectos de uma determinada atividade empresarial, que são fundamentais para $\circ$ entendimento da política de sustentabilidade da empresa (Ferguson \& Pündrich, 2015) e para oferta de serviços de asseguração de alta qualidade (Martínez-Ferrero et al., 2018). Então, se um determinado provedor de garantia é especialista 
em um determinado setor, as empresas desse setor terão uma maior propensão a contratá-lo, com a expectativa de obter uma maior qualidade de serviço (Fernandez-Feijoo et al., 2016).

Martínez-Ferrero e García-Sánchez (2018), ao examinarem o impacto da reputação e da especialização do setor dos provedores no trabalho da asseguração dos $R S$, concluíram que a probabilidade de detectar erros e omissões materiais em um RS é maior se for verificada por uma Big-4 e por um especialista do setor. A experiência na prestação de serviços de auditoria e as habilidades e treinamentos relevantes fornecidos pelas firmas Big-4, bem como o conhecimento e a experiência de especialistas do setor, aumentam a propensão de relatar opiniões mais precisas sobre os RS. Consistente com esses argumentos, estabelece-se a segunda hipótese de pesquisa.

$\mathrm{H} 2$ : A presença das grandes firmas de contabilidade (Big-4) no mercado de asseguração externa dos RS é afetada positivamente quando o provedor contratado for especializado no setor da empresa relatora.

Considerando a perspectiva da racionalidade coletiva, a decisão das empresas sobre qual provedor contratar pode ser motivada por mecanismos informais que possibilitam reforçar a sua reputação social (Fernandez-Feijoo et al., 2016). Além disso, Pflugrath et al. (2011) constataram uma maior credibilidade, percebida pelos investidores, para as assegurações realizadas por profissionais de contabilidade, tipicamente atribuídos a firmas Big-4. Nesse quesito, devido à experiência no fornecimento de serviços tradicionais de auditoria, as principais firmas de contabilidade são percebidas como experts nos trabalhos de asseguração dos RS (Farooq \& De Villiers, 2019).

O efeito "marca" ou capital reputacional das firmas Big4 pode ser considerado ou percebido como suficiente para transferir credibilidade aos RS (O'Dwyer \& Owen, 2005; Hummel et al., 2017; Martínez-Ferrero \& GarcíaSánchez, 2018). O reconhecimento das grandes firmas de auditoria no campo da contabilidade e das suas redes de negócios bem estabelecidas aumentam sua legitimidade e seu domínio sobre o mercado de asseguração dos RS (Perego, 2009; Kolk \& Perego, 2010; FernandezFeijoo et al., 2016). Nesse sentido, Boiral et al. (2020) apontaram que a natureza altamente profissionalizada e estruturada da auditoria financeira em termos de normas, formação, reconhecimento externo, códigos de conduta e competências é utilizada como uma espécie de rótulo de qualidade para legitimar a atividade das grandes firmas de contabilidade nesse mercado.
Possíveis explicações poderiam ser fornecidas pela teoria da legitimidade, desde que as empresas também enfatizam o contexto social no qual operam (Bansal, 2005) e são susceptíveis de se esforçar por mais legitimidade ao demonstrar para o público, que podem confiar no conteúdo divulgado em seus relatórios (Hummel et al., 2017). Nessa lógica, a pressão social (Alrazi et al., 2015) pode ser razão subjacente para que as firmas Big-4 atuem no mercado de asseguração externa dos RS, pois as empresas podem estar sujeitas a um ambiente de legitimação. E a legitimidade organizacional pode resultar de comportamentos isomórficos ou miméticos quando uma prática é amplamente adotada em um mesmo setor ou país (Meyer \& Rowan, 1977).

DiMaggio e Powell (1983) acrescentam que comportamentos miméticos resultam da incerteza, uma força que incentiva a imitação. Em contextos emergentes, como o mercado da asseguração externa dos RS, no qual paira a dúvida sobre qual provedor competente contratar, é possível que esses mecanismos miméticos levem as empresas, em um contexto específico, a se comportarem de maneira semelhante em relação à escolha do provedor (Fernandez-Feijoo et al., 2016).

Sierra-García et al. (2013) sugeriram que a decisão de contratar um auditor contábil para assegurar os RS pode estar relacionada ao setor. A respeito disso, Peters e Romi (2014) encontraram evidências de que as empresas se tornam semelhantes em suas práticas de asseguração quando tais práticas são amplamente adotadas em seu setor.

Como se verifica, se um provedor de garantia possui uma reputação elevada em determinado setor, é provável que essa reputação motive outras empresas a contratá-lo, o que permite a formulação da terceira e última hipótese de pesquisa:

H3: A presença das grandes firmas de contabilidade (Big4) no mercado de asseguração externa dos relatórios de sustentabilidade é afetada positivamente pelo isomorfismo mimético.

Embora os atributos das firmas Big-4 sugiram possíveis benefícios para o processo de asseguração externa dos RS, a natureza discricionária e desregulamentada da asseguração externa RS permite que as empresas relatoras controlem os aspectos mais críticos do processo, por exemplo: a escolha do provedor, o escopo da auditoria e o acesso às informações (Boiral et al., 2019). 
Nesse aspecto, a independência é fundamental para garantir a confiança do público nas informações de sustentabilidade por se referir à capacidade de o provedor fornecer uma opinião imparcial sobre o RS (Boiral \& Heras-Saizarbitoria, 2020). Todavia, devido aos interesses econômicos e profissionais de inserção neste mercado, os provedores podem ser persuadidos a dar prioridade à satisfação do cliente sobre o ceticismo profissional e a verificação imparcial (Boiral et al., 2020; Ruiz-Barbadillo \& Martínez-Ferrero, 2020).

Nota-se que, muitas vezes, a asseguração externa do RS tende a ser conduzida pelos interesses e objetivos das empresas e não pela demanda das demais partes interessadas por informações mais transparentes e confiáveis (O'Dwyer \& Owen 2005; Boiral et al., 2019). $E$, frequentemente, as empresas preocupam-se em buscar uma opinião independente acerca de seus RS para fins de gerenciamento de imagem e não para melhorar seus pontos fracos relativos à sustentabilidade (Jones \& Solomon, 2010; Hummel et al., 2017).

\section{Procedimentos Metodológicos}

\subsection{Seleção da amostra e dados coletados}

Este estudo, inicialmente, examinou o total de 391 companhias listadas na Brasil, Bolsa e Balcão (B3) em 2019 para identificar quais empresas asseguraram externamente os RS. A amostra compreende empresas que publicaram as informações de sustentabilidade asseguradas no formato stand-alone (publicação de um relatório independente), os quais estão disponíveis em websites e possuem dados necessários para operacionalizar as variáveis do estudo no período temporal de 2012 a 2018.

O recorte amostral e a seleção do espaço de temporal de análise tiveram como referência o "Comunicado Externo 017/2011-DP" da B3, o qual orienta que as empresas listadas relatem, a partir de 2012, em seu Formulário de Referência (FR) (item 7.8 - Políticas Socioambientais), se realizam a divulgação e a asseguração externa das informações de sustentabilidade, e, em caso negativo, sua justificativa.

Destaca-se que os RS não seguem padrões regulares de divulgação e, por isso, o período de análise findou em 2018, já que, até o momento da coleta (outubro de 2019 a março de 2020), somente uma empresa havia publicado o relatório referente ao ano de 2019.

Após a identificação das empresas, optou-se por excluir 8 empresas que pertenciam aos setores de petróleo (1), saúde (2) e telecomunicações (2) em todos os anos de análise e consumo cíclico (3) entre os anos de 2012-2016, uma vez que a baixa representatividade dificulta identificar provedores especialistas nesses setores. A esse respeito, a empresas foram classificadas de acordo com a lista de segmentos da B3 (descritos na subseção 4.1 deste trabalho) e considerouse como representatividade a quantidade mínima de três (3) empresas por setor.

No geral, a quantidade de companhias participantes da pesquisa foi de 47 empresas. No entanto, a composição da amostra não foi a mesma para todos os anos. Observando a discricionariedade da asseguração externa dos RS, a análise contou com um conjunto de dados em painel desbalanceado, resultante em 249 observações, pois nem todas as empresas da amostra contrataram os serviços de asseguração externa em todos os anos.

As informações sobre a natureza dos provedores de asseguração foram obtidas pelo exame dos RS do website de cada empresa. Já os dados relativos à prestação de serviços de auditoria contábil Big-4 foram extraídos das demonstrações contábeis disponibilizadas no website da B3. Por fim, as informações contábeis e financeiras (ativos totais, ROA e passivos) foram coletadas do banco de dados da Economática ${ }^{\circledR}$.

\subsection{Definição das variáveis de estudo}

A variável dependente $\mathrm{BIG} 4$ assumida consiste em uma variável binária ou nominal codificada como 0 e 1 (dummy). Dessa forma, quando a empresa relatora contrata uma firma Big-4 (KPMG, PWC, Deloitte e Ernst \& Young) como provedora da asseguração externa de seu RS é atribuído 1, e para os casos em que as empresas não contrataram provedor dessa natureza, atribui-se 0 . Esta é uma medida comum na literatura para avaliar os fatores que influenciam a decisão das empresas em contratar firmas de contabilidade para assegurar seus RS (Simnett et al., 2009; Fernandez-Feijoo et al., 2016; Martínez-Ferrero \& García-Sánchez, 2018). As informações acerca da natureza dos provedores foram averiguadas nos relatórios de asseguração externa anexos aos RS.

Para compreender quais atributos relacionados ao capital reputacional dos provedores contábeis podem afetar - evento de interesse de pesquisa, foram testadas três variáveis independentes, fundamentadas mediante pesquisas anteriores, que representam a prestação conjunta de serviços de auditoria contábil e asseguração dos RS $(\mathrm{H} 1)$, especialização no setor $(\mathrm{H} 2)$ e isomorfismo mimético $(\mathrm{H} 3)$. 
Além disso, foram utilizadas as variáveis de controle Tamanho (TAM); Rentabilidade (RENT) e Endividamento (ENDIV). Argumenta-se que empresas maiores e mais rentáveis tendem a ter recursos financeiros necessários para promover atividades ligadas à sustentabilidade, já que a assegurar voluntariamente os RS acarreta custos adicionais para as empresas. A Tabela 1 demonstra as variáveis independentes e como estas foram fundamentas e mensuradas.

Tabela 1: Definições das variáveis independentes da pesquisa

\begin{tabular}{|c|c|c|}
\hline Variável & Mensuração & Fundamentação \\
\hline $\begin{array}{c}\text { Prestação conjunta de } \\
\text { serviços de auditoria } \\
\text { contábil e asseguração } \\
\text { dos RS } \\
\text { (AUDIT_ASSURANCE) }\end{array}$ & $\begin{array}{l}\text { Variável dummy que } \\
\text { assume valor } 1 \text { se a } \\
\text { Big-4 " } X \text { " que assegurou } \\
\text { o RS também auditou as } \\
\text { demonstrações contábeis } \\
\text { e, } 0 \text { caso contrário. }\end{array}$ & $\begin{array}{l}\text { Park e Brorson (2005); Jones } \\
\text { e Solomon (2010); Gillet } \\
\text { (2012); Byus et al.'(2013) } \\
\text { e Fernandez-Feijoo et al. } \\
\text { (2016). }\end{array}$ \\
\hline $\begin{array}{l}\text { Especialização no setor } \\
\text { (ESPEC) }\end{array}$ & $\begin{array}{l}\text { Variável dummy que } \\
\text { assume o valor } 1 \text { quando } \\
\text { asseguração é confiada } \\
\text { a um especialista do } \\
\text { setor como provedor e, } 0 \\
\text { caso contrário. }\end{array}$ & $\begin{array}{l}\text { Casterella et al. (2004); } \\
\text { Chen, Lin e Zhou, (2005); } \\
\text { Fernandez-Feijoo et al. } \\
\text { (2016) e Martínez-Ferrero e } \\
\text { García-Sánchez (2018). }\end{array}$ \\
\hline $\begin{array}{l}\text { Isomorfismo mimético } \\
\text { (ISOMORPH) }\end{array}$ & $\begin{array}{l}\text { Quantidade de empresas } \\
\text { do mesmo setor que } \\
\text { contrataram o mesmo } \\
\text { provedor para assegurar } \\
\text { seu RS. }\end{array}$ & $\begin{array}{c}\text { Sierra-García et al., (2013); } \\
\text { Peters e Romi (20 14) e } \\
\text { Fernandez-Feijoo et al. } \\
(2016) .\end{array}$ \\
\hline $\begin{array}{l}\text { Tamanho } \\
\text { (TAM) }\end{array}$ & Logaritmo do ativo total. & \multirow{3}{*}{$\begin{array}{l}\text { Simnett et al., } 2009 ; \\
\text { Hummel et al., } 2017^{\prime} \text { e } \\
\text { Martínez-Ferrero e García- } \\
\text { Sánchez, 2018. }\end{array}$} \\
\hline $\begin{array}{l}\text { Rentabilidade } \\
\text { (RENT) }\end{array}$ & $\begin{array}{l}\text { Retorno sobre os Ativos } \\
\text { (ROA). }\end{array}$ & \\
\hline $\begin{array}{l}\text { Endividamento } \\
\text { (ENDIV) }\end{array}$ & $\begin{array}{l}\text { Soma do Passivo } \\
\text { Circulante e Não } \\
\text { Circulante divido pelo } \\
\text { Ativo Tołal. }\end{array}$ & \\
\hline
\end{tabular}

Fonte: Dados da pesquisa.

Destaca-se que, apesar de a especialização no setor (ESPEC) não ser diretamente observável nos estudos da asseguração externa dos RS, a literatura anterior sobre auditoria financeira empregou diversas proxies para mensurá-la. A maioria dessas proxies baseia-se na participação de mercado. Devido à disponibilidade dos dados, este trabalho utilizou, como base de mensuração para a participação de mercado do provedor de asseguração em um determinado setor, o valor do ativo dos clientes (Chen et al., 2005), que consiste na razão entre a soma do ativo total de todos os clientes de um determinado provedor que pertencem ao mesmo setor e a soma do ativo total de todas as empresas do respectivo setor.

O especialista do setor é aquele que detém notável atuação em um determinado setor. Dessa forma, o estudo utilizou a margem de $20 \%$ das quotas de mercado para caracterizar determinado provedor como especialista (Craswell et al., 1995; Casterella et al., 2004; Chen et al., 2005) Ressalta-se que, em função da competição de diferentes provedores no mercado de asseguração externa dos RS, a especialização do setor não é uma característica restrita a firmas Big-4. Por isso, esta pesquisa considerou como possíveis especialistas do setor tanto as firmas de auditoria contábil Big-4 quanto os demais provedores atuantes nesse mercado.

A variável isomorfismo mimético (ISOMORPH) presume que uma empresa pode contratar uma firma Big-4 para assegurar seus RS para imitar e/ou se assemelhar ao comportamento dos demais participantes de um mesmo ambiente social. Desse modo, adota-se a medida proposta por Peters e Romi (2014) que representa a quantidade de empresas do mesmo setor que contrataram o mesmo provedor para assegurar seu RS.

\subsection{Técnica de Análise dos dados}

A técnica estatística utilizada consistiu em um modelo estatístico de regressão hierárquico logístico para dados em painel, visto que esta pesquisa objetivou estimar a probabilidade de uma empresa contratar uma firma Big-4 como provedor de asseguração externa dos RS no período de 2012-2018. Destaca-se que esse evento pode ocorrer ou não; afinal, diversos tipos de provedores atuam nesse mercado, em função das variáveis explicativas inseridas no modelo.

Segundo Hox (2010), os modelos hierárquicos estimam, com eficiência, o erro padrão dos coeficientes de regressão, pois consideram a suposição de que as observações dentro de um conglomerado podem estar correlacionadas. Dessa maneira, o emprego desse modelo é recomendado para amostras com dados aninhados ou com medidas repetidas. Ademais, o modelo possibilita que determinados dados de uma empresa, que não variam ao longo do tempo, possam ser comparados com dados de outra empresa, permitindo que o pesquisador avalie as diferentes influências sobre o comportamento da variável dependente (Fávero \& Belfiore, 2017). A estrutura de dados com medidas repetidas para a amostra dessa pesquisa foi definida em dois níveis, considerando as variáveis independentes da empresa i que "variam no tempo" (nível 1 - tempo) e as variáveis independentes da empresa i "fixas no tempo" (nível 2 - empresa).

A Equação 1 descreve o modelo de regressão hierárquico logístico para a análise de dados em painel utilizado para testar as hipóteses de pesquisa

$$
\begin{aligned}
& \log \left[\text { prob(BIG4it) } /(1-\operatorname{prob}(B \mid G 4 i t)]=a_{i}+\beta_{1} A U D F I N_{i t}+\beta_{2}\right. \\
& \text { ESPEC }_{\mathrm{it}}+\beta_{3} \text { REPUT }_{\mathrm{itt}}+\beta_{4} \text { TAM }_{\mathrm{it}}+\beta_{5} \text { RENT }_{\mathrm{it}}+\beta_{6} \text { ENDIV }_{\mathrm{it}}+\mu_{\mathrm{Ot}} \text { (1) }
\end{aligned}
$$

BIG4it representa a variável dependente dummy, que assume: 1 quando a asseguração externa dos RS é realizada por uma firma de contabilidade Big-4; e 0, caso contrário. Além disso, $a_{i}$ é o intercepto da equação; $\beta_{1^{\prime}} \beta_{2^{\prime}} \beta_{3^{\prime}} \beta_{4^{\prime}} \beta_{5^{\prime}}$ $\beta_{6^{\prime}}$ e $\beta_{7}$ os receptivos parâmetros estimados com as seguintes 
variáveis explicativas: Prestação conjunta de serviços de auditoria contábil e asseguração dos RS, Especialização no Setor, Isomorfismo mimético, Tamanho, Rentabilidade e Endividamento; e $\mu_{0 \dagger}$ o termo aleatório da regressão.

Os resultados da regressão descrita acima e de testes adicionais, como a análise de correspondência (Anacor) e a análise de homogeneidade (HOMALS), foram obtidos por meio da utilização dos softwares estatísticos: $R$ versão 3.6.3 for Windows, pacotes "Ime4" e "nIme", e Stata ${ }^{\circledR}$ 15.A respeito dos testes adicionais, a Tabela 2 demonstra a categorização e os níveis de associação atribuídos para cada variável de interesse utilizada para caracterizar as atuações individuais de cada uma das Big-4 de acordo com a prestação conjunta de serviços de auditoria contábil e asseguração dos RS e setor de atividade da empresa relatora.

Tabela 2: Categorização e níveis de associação das variáveis

\begin{tabular}{|c|c|c|}
\hline Variáveis & Definição & $\begin{array}{c}\text { Nível de } \\
\text { associaçãão }\end{array}$ \\
\hline \multirow{2}{*}{ BIG4 } & \multirow{2}{*}{$\begin{array}{l}\text { A Big-4 "X" (KPMG ou PWC ou Deloitte ou } \\
\text { Ernest \& Young) é o provedor contratado } \\
\text { para garantir o RS. }\end{array}$} & Contratado \\
\hline & & Não contratado \\
\hline \multirow{2}{*}{ AUDFIN } & \multirow{2}{*}{$\begin{array}{l}\text { A Big-4 " "X" que auditou as demonstrações } \\
\text { contábeis também foi a responsável pela } \\
\text { asseguração do RS. }\end{array}$} & Contratado \\
\hline & & Não contratado \\
\hline \multirow[t]{2}{*}{ SETOR } & \multirow{2}{*}{$\begin{array}{l}\text { Ramo de atividade que as empresas atuam } \\
\text { no mercado de açôes brasileiro, as quais } \\
\text { foram classificadas conforme a listagem da } \\
\text { B3: 1- Utilidade Pública, 2- Consumo não } \\
\text { Cíclico, 3- Consumo Cíclico, 4- Materiais } \\
\text { Básicos, 5- Bens Industriais e 6- Financeiro. }\end{array}$} & Pertence \\
\hline & & Não pertence \\
\hline
\end{tabular}

Fonte: Dados da pesquisa.

Destaca-se que a Anacor retrata a correspondência de categorias de variáveis qualitativas, e esta correspondência é a base para 0 desenvolvimento de mapas perceptuais que permite visualizar medidas de distância em um espaço bidimensional. Nessa técnica, o teste Qui-quadrado é empregado para avaliar a existência de associações significativas entre as variáveis de pesquisa a partir de uma tabela de contingência que calcula as diferenças entre as frequências esperadas e observadas (Fávero \& Belfiore, 2017). Para representação visual dos mapas perceptuais das associações entre as variáveis, utilizou-se a análise de HOMALS. Na Figura 1, apresenta-se o modelo teórico da pesquisa, o qual destaca a influência dos atributos relacionados ao capital reputacional das grandes firmas de auditoria contábil na sua participação no mercado da asseguração dos RS.

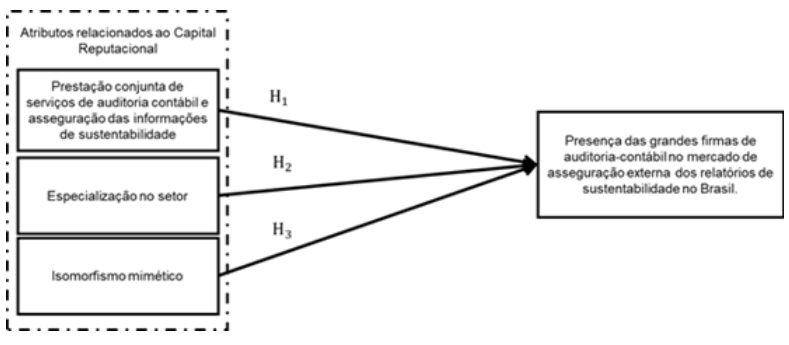

Figura 1. Modelo teórico da pesquisa

Fonte: Elaboração própria.

Especificamente, este artigo argumenta que os efeitos positivos da prestação conjunta de serviços de auditoria contábil e asseguração dos RS, especialização no setor e isomorfismo mimético na contratação das grandes firmas de auditoria contábil como provedor da asseguração externa dos RS atestam o domínio, "tenure", desses profissionais neste emergente mercado.

\section{Resultados e Discussões}

\subsection{Estatísticas descritivas e teste das hipóteses da pesquisa}

O levantamento dos dados contemplou o exame de 249 relatórios de sustentabilidade assegurados entre o período de 2012-2018 em 6 distintos setores da B3. A distribuição da amostra de acordo com o provedor e o setor é discriminada na Tabela 3.

Tabela 3: Distribuição da amostra de acordo com o provedor e o setor

\begin{tabular}{|c|c|c|c|c|c|c|}
\hline Setor & KPMG & PWC & EY & Deloitte & Outros & $\%$ Big-4 total \\
\hline Utilidade Pública & 38 & 11 & 3 & 7 & 16 & 78,67 \\
\hline $\begin{array}{c}\text { Consumo não } \\
\text { Cíclico }\end{array}$ & 10 & 1 & 9 & - & 8 & 71,43 \\
\hline \begin{tabular}{c} 
Consumo Cíclico \\
\hline $\begin{array}{c}\text { Materiais } \\
\text { Básicos }\end{array}$
\end{tabular} & 11 & - & - & - & 3 & 57,14 \\
\hline $\begin{array}{c}\text { Bens Industriais } \\
\text { Financeiro }\end{array}$ & 9 & 1 & 6 & 5 & 7 & 75,00 \\
\hline Total & 103 & 33 & 26 & 25 & 62 & 187 \\
\hline \% sobre Total & 41,37 & 13,25 & 10,44 & 10,04 & 24,09 & - \\
\hline
\end{tabular}

Legenda: \% = Percentual.

Fonte: Dados da pesquisa. 
Nota-se que as empresas de auditoria Big-4 possuem uma participação de 75,1\% (187/249) nos serviços asseguração dos RS realizados. De forma geral, KPMG e PWC se destacam, com um percentual de 41,37\% (103/249) e $13,25 \%$ (33/249), respectivamente. Quanto à atuação dos provedores por setor, verifica-se que as Big-4 estiveram presentes em mais de $50 \%$ na maioria dos setores da amostra, enquanto os provedores não Big-4 (Outros) obtiveram a maior participação de mercado apenas no setor de materiais básicos. A KPMG, consideravelmente, foi a Big-4 de maior atuação em todos os setores, com destaque para os setores de utilidade pública e financeiro.

A representação visual apresentada na Figura 2 caracteriza as atuações individuais de cada Big-4 de acordo com a prestação conjunta de serviços de auditoria contábil e asseguração dos RS (Qui-quadrado $=58,67, p>0,05)$ e setor de atividade da empresa relatora (Qui-quadrado $=110,02$, p >0,05) no período de 2012-2018.

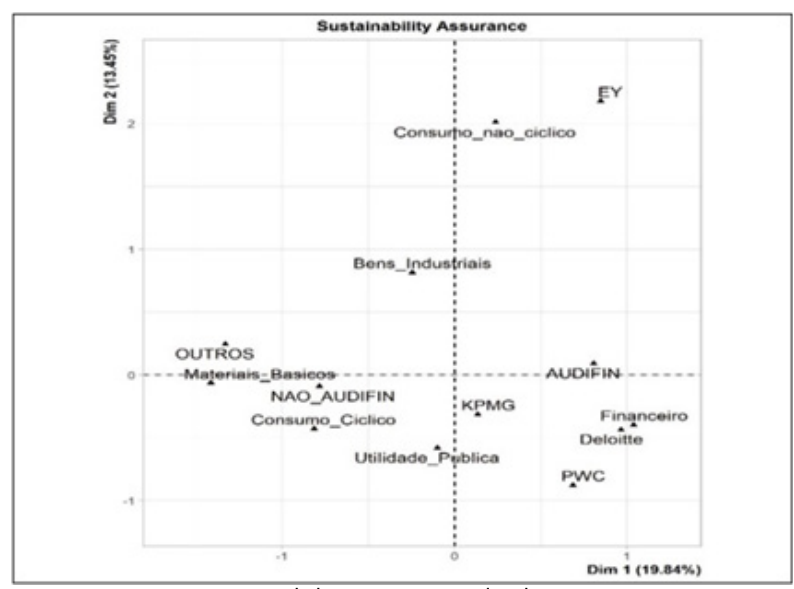

Figura 2. Mapa perceptual das atuações individuais por Big-4

Legenda: NAO AUDIFIN = Não é auditor das demonstrações contábeis: AUDIFIN = auditor das demonstrações contábeis.

Fonte: Dados da pesquisa.

Quanto à distribuição por setor, destaca-se que o financeiro está mais associado a contratar a prestação conjunta de serviços de auditoria contábil e asseguração dos RS, sendo a Deloitte a Big-4 mais próxima. O setor de utilidade pública se mostrou mais associado a Big-4 KPMG, PWC e Deloitte; porém, menos direcionado a contratar o auditor das demonstrações contábeis do que o setor financeiro. Consumo cíclico, materiais básicos e bens industriais são os setores mais associados à contratação de profissionais não Big-4, apesar de consumo cíclico e bens industriais manterem-se próximas da KPMG. Já o setor de consumo não cíclico está mais associado a Ernst \& Young. Além disso, os setores de consumo cíclico e materiais básicos são os que mais se distanciam de tomar o auditor contábil como provedor.
Observa-se, ainda, que a Deloitte, KPMG e PWC estão mais associadas a utilizar sua rede de clientes de auditoria contábil para entrar no mercado da asseguração externa dos RS do que a Ernst \& Young. Por outro lado, os provedores não Big-4 "Outros", predominantemente compostos por empresas de consultorias de engenharia, certificações e especialistas em sustentabilidade, estão mais associados a não serem auditores das demonstrações contábeis e financeiras, uma vez que somente profissionais de contabilidade são habilitados para essa função.

A Tabela 4 descreve a frequência dos auditores das demonstrações contábeis e especialistas como provedores de asseguração externa dos RS.

Tabela 4: Frequências relativas das variáveis qualitativas

\begin{tabular}{|c|c|c|}
\hline Variáveis qualitativas & Frequência & $\%$ \\
\hline BIG4 & & \\
\hline 0 & 62 & 24,90 \\
\hline 1 & 187 & 75,10 \\
\hline AUDIT_ASSURANCE & & \\
\hline 0 & 126 & 50,60 \\
\hline 1 & 123 & 49,40 \\
\hline ESPEC & & 39,76 \\
\hline 0 & 99 & 60,24 \\
\hline 1 & 150 & $\%$ \\
\hline Provedor - Especialista do setor & Frequência & 60,67 \\
\hline KPMG & 91 & 12,67 \\
\hline PWC & 19 & 4,00 \\
\hline EY & 6 & 3,33 \\
\hline Deloitte & 5 & 19,33 \\
\hline Outros & 29 & $c 0 n$ \\
\hline
\end{tabular}

Legenda: $\%$ = Percentual; AUDIT_ASSURANCE = Prestação conjunta de serviços de auditoria contábil e asseguração dos RS; ESPEC = Especialização no setor.

Fonte: Dados da pesquisa.

De acordo com a Tabela 4, quase a metade dos RS da amostra $(49,4 \%)$ foi assegurada pela mesma firma de auditoria Big4 que auditou a demonstrações contábeis, o que representa $65,77 \%$ do total das assegurações realizadas pelas Big-4. Das assegurações contratadas, $60,24 \%$ correspondem aos trabalhos realizados por profissionais especialistas no setor. Desses profissionais, a KPMG é a empresa Big-4 com mais especializações, sendo especialista nos seguintes setores: utilidade pública (todos os anos), consumo não cíclico (20132018), consumo cíclico (2014-2018), materiais básicos (2012), bens industriais (2012, 2013, 2015, 2017 e 2018) e financeiro (todos os anos). A PWC é a segunda empresa Big-4 com mais especializações, para os setores: consumo cíclico (2014), bens industriais (2014) e financeiro (todos os anos); seguida pela Ernst \& Young com especializações 
nos setores de consumo não cíclico (2016 e 2018), consumo cíclico (2012 e 2013), bens industriais (2013, 2014 e 2015). Por último, a Big-4 Deloitte representa 3,33\% do total de especializações identificadas somente para o setor de bens industriais $(2012,2014,2015,2016$ e 2018).

Ainda, sobre a especialização para as empresas não Big-4, os provedores "Outros" corresponde a 19,33\% do total das especializações, sendo especialista nos setores de utilidade pública (2012, 2016, 2017 e 2018), consumo não cíclico (2012), consumo cíclico (2012 e 2013) e materiais básicos (łodos os anos).

$\mathrm{Na}$ Tabela 5, são relatadas as estatísticas descritivas juntamente com a matriz de correlação entre as variáveis de interesse. As estatísticas univariadas suportam os dados anteriores da Tabela 5, indicando que empresas de auditoria Big-4 dominaram o mercado brasileiro da asseguração externa dos RS com um percentual de $75,1 \%$.

Tabela 5: Estatísticas descritivas e matriz de correlação

\begin{tabular}{|c|c|c|c|c|c|c|c|c|c|}
\hline Variáveis & Média & DP & 1 & 2 & 3 & 4 & 5 & 6 & 7 \\
\hline 1. BIG4 & 0,751 & 0,433 & 1 & & & & & & \\
\hline $\begin{array}{c}\text { 2. AUDIT } \\
\text { ASSURANCE }\end{array}$ & 0,493 & 0,500 & $0,439^{* *}$ & 1 & & & & & \\
\hline 3. ESPEC & 0,602 & 0,490 & $0,158^{*}$ & $-0,0344$ & 1 & & & & \\
\hline $\begin{array}{c}4 . \\
\text { ISOMORPH }\end{array}$ & 2,726 & 1,759 & $0,340^{* *}$ & $0,1435^{*}$ & $0,5469^{* *}$ & 1 & & & \\
\hline 5.TAM & 17,089 & 1,717 & $0,136^{*}$ & $0,1471^{*}$ & $0,3665^{* *}$ & $0,2461^{* *}$ & 1 & & \\
\hline 6. ENDIV & 0,698 & 0,208 & 0,120 & $0,3451^{* *}$ & 0,0039 & $-0,037$ & $0,2841^{* *}$ & 1 & \\
\hline 7. RENT & 0,032 & 0,064 & $0,246^{* *}$ & 0,0133 & 0,0199 & 0,0336 & $-0,1277^{*}$ & $-0,4347^{* *}$ & 1 \\
\hline
\end{tabular}
Legenda: DP = Desvio Padrão; AUDIT_ASSURANCE = Prestação conjunta de serviços de auditoria contábil e asseguração dos RS; ESPEC = Especialização no setor; ISOMORPH = Isomorfismo mimético; TAM = Tamanho; ENDIV = Endividamento; RENT = Rentabilidade.

Nota: * e correspondem a $5 \%$ e $1 \%$ de nível de significância, respectivamente.

Fonte: Dados da pesquisa.

O valor médio da variável ISOMORPH, que se refere à quantidade de empresas do mesmo setor que contrataram o mesmo provedor de garantia, indica que 2,72 dos RS são assegurados pelo mesmo provedor. Em média, 3,09 relatórios, relatados por empresas do mesmo setor durante o período de 2012-2018, foram assegurados por uma Big-4, enquanto os provedores não Big-4 obtiveram a média 1,65. A KPMG assegurou, em média, 4,07 relatórios de empresas do mesmo setor. Ao passo que PWC, Ernst \& Young e Deloitte apresentaram médias de 2,21, 1,46 e 1,88, respectivamente.

Por meio da matriz de correlação, exibida na Tabela 6, verifica-se que não há valores altos para os coeficientes entre variáveis dependentes e independentes ou entre variáveis independentes, considerando que as correlações se apresentam, em sua maioria, como moderadas $(0,40$ a $0,69)$ e baixas $(0,01$ a 0,39$)$, e isso indica que o modelo não apresenta problemas de multicolinearidade.

O Teste de Hosmer-Lemeshow $=37,71$ (p-valor de 0,7711) indicou evidências de associação e considera que o modelo apresenta um bom ajuste. $O$ modelo logístico classificou $77,51 \%$ das observações analisadas $([159+34] / 249=$ $0,7751)$ e obteve capacidade discriminatória suficiente (curva ROC $=0,8791$ ). Os resultados da regressão logística em painel de efeito mistos estão resumidos na Tabela 6.

Tabela 6: Resultados da regressão logística em painel de efeito mistos

\begin{tabular}{|c|c|c|c|c|c|c|}
\hline \multirow{5}{*}{ BIG4 } & \multicolumn{6}{|c|}{ Efeitos Aleatórios } \\
\hline & Grupos & Nome & Variância & \multicolumn{3}{|c|}{ Desvio Padrão } \\
\hline & Empresas & Constante & 23,08 & \multicolumn{3}{|c|}{4,805} \\
\hline & \multicolumn{6}{|c|}{ Efeitos Fixos } \\
\hline & Odds Ratio & Coeficiente & $\begin{array}{l}\text { Erro } \\
\text { Padrão }\end{array}$ & z-valor & p-valor & Significância \\
\hline Constante & 0,0003 & $-8,0667$ & 11,3201 & $-0,713$ & 0,4761 & \\
\hline $\begin{array}{c}\text { AUDIT } \\
\text { ASSURANCE }\end{array}$ & 182,1524 & 5,2048 & 2,0852 & 2,496 & 0,0126 & $*$ \\
\hline ESPEC & 0,4212 & $-0,8646$ & 1,0518 & $-0,822$ & 0,4110 & \\
\hline ISOMORPH & 3,1589 & 1,1502 & 0,4741 & 2,426 & 0,0153 & $*$ \\
\hline TAM & 1,5099 & 0,4121 & 0,6900 & 0,597 & 0,5504 & \\
\hline ENDIV & 2,4370 & 0,8908 & 4,5346 & 0,196 & 0,8443 & \\
\hline RENT & $7.502 .123,00$ & 15,8307 & 9,0247 & 1,754 & 0,0794 & \\
\hline Observaçōes & \multicolumn{5}{|c|}{249} & \\
\hline Log likelihood & \multicolumn{5}{|c|}{$-55,6$} & \\
\hline Deviance & \multicolumn{5}{|c|}{111,2} & \\
\hline $\mathrm{BIC}$ & \multicolumn{5}{|c|}{155,3} & \\
\hline
\end{tabular}

Legenda: AUDIT_ASSURANCE = Prestação conjunta de serviços de auditoria contábil e asseguração dos RS; ESPEC = Especialização no setor; ISOMORPH = Isomorfismo mimético; TAM = Tamanho; ENDIV = Endividamento; RENT = Rentabilidade. BIC é o critério de informação bayesiano, com base em - 2 log de pseudo verossimilhança.

Nota: * estatisticamente significante a 95\% de confiança.

Fonte: Dados da pesquisa

Os resultados descritos na Tabela 6 sugerem que a probabilidade de uma firma Big-4 ser escolhida para assegurar os RS é afetada positivamente pelo fato de que - mesmo Big-4 também é o auditor das demonstrações contábeis $(5,2048, p<0,05)$, bem como pela sua reputação elevada em um determinado setor $(1,1502, p<0,05)$. A razão de chances ou coeficiente exponencial (Odds Ratio) indica que as chances de escolher uma Big-4 como provedor é aproximadamente 182 vezes maior nas empresas em que essa Big-4 também audita as demonstrações contábeis. Quanto à ISOMORPH, que reflete a quantidade de empresas do mesmo setor que contrataram o mesmo provedor, aumentase em torno de três vezes a probabilidade de uma Big-4 assegurar o RS quando esse provedor atua com notoriedade 
em um contexto específico, como setor.

\subsection{Discussão dos Resultados}

Os resultados desse estudo apontam para o predomínio das firmas de auditoria contábil Big-4 em relação aos demais provedores, visto que tais firmas são responsáveis por $75,1 \%$ dos trabalhos realizados e atuantes em mais de $50 \%$ na maioria dos setores da amostra. Este estudo sugere que esse domínio pode estar relacionado não somente com a probabilidade de as empresas brasileiras contratarem a firma de auditoria Big-4 que auditou suas demonstrações contábeis para assegurar seus RS como também ao fato de esse tipo de profissional ser percebido como provedor legítimo em determinados contextos sociais, o que, nesta pesquisa, se refere ao setor no qual a empresa opera.

A significância estatística do coeficiente da variável AUDIT_ASSURANCE, que se refere a hipótese $\mathrm{H} 1(5,2048$, $p<0,05)$, indica que as empresas Big-4 encontram, na sua rede de clientes de auditoria financeira, uma oportunidade de fornecer serviços de asseguração externa dos RS. Esse resultado reforça a conclusão de Fernandez-Feijoo et al. (2016) sobre a potencial vantagem competitiva das Big-4, uma vez que essa estratégia fomenta seu engajamento em contratos de asseguração e adiciona barreiras à participação de outros provedores nesse mercado.

Possíveis explicações são apontadas na literatura para a vantagem competitiva das firmas Big-4 no mercado da asseguração externa dos RS. Há a perspectiva de que as empresas consideram que $\circ$ auditor das demonstrações contábeis detém conhecimentos sobre suas operações e experiência em trabalhos de auditoria necessários para a realização do trabalho de garantia, os quais permitem economia de escopo, redução de taxas de asseguração e maior qualidade do trabalho de asseguração (Jones \& Solomon, 2010; Gillet, 2012; Martínez-Ferrero \& GarcíaSánchez, 2018).

Por outro lado, a dependência econômica e o vínculo com a empresa relatora podem ser importantes razões subjacentes dessa relação (Boiral et al., 2020; Ruiz-Barbadillo \& MartínezFerrero, 2020). Sobre esse aspecto, é preciso refletir que a falta de independência afeta a capacidade de o provedor realizar o trabalho de maneira objetiva, comprometendo a qualidade do trabalho realizado, o que resultaria em uma opinião distorcida para os usuários do RS (Farooq \& de Villiers, 2017). Esse fato parece sugerir questionamentos acerca do valor agregado aos trabalhos de asseguração externa dos RS. Além disso, importantes implicações éticas a respeito da conduta corporativa e dos profissionais que atuam nesse mercado surgem se esses serviços forem utilizados como estratégia empregada no gerenciamento de imagem (Jones \& Solomon, 2010; Hummel et al., 2017).

Semelhante a Martínez-Ferrero e García-Sánchez (2018) e Fernandez-Feijoo et al. (2016), esta pesquisa identificou a ocorrência de especializações para as firmas Big-4. Em suma, há predominância da KPMG nos setores de utilidade pública e financeiro, PWC no setor financeiro, e finalmente Deloitte e Ernst \& Young no setor de bens industriais. Entretanto, os resultados fornecidos pela análise de regressão não suportam evidências de que a especialização no setor (H2), ESPEC, observada sob aspecto de tamanho e complexidade do cliente (Chen et al., 2005), favoreça a presença das Big-4 no mercado da asseguração externa dos RS $(-0,8646, p=$ $0,4110)$.

Por outro lado, esta pesquisa argumentou que as Big-4 podem ser consideradas experientes em um determinado campo a partir de sua reputação na percepção da maioria das empresas de um determinado setor (ISOMORPH), referente a hipótese H3 $(1,1502, p<0,05)$. Desse modo, a decisão de contratar uma Big-4 para assegurar os RS pode estar relacionada aos mecanismos informais, como o reforço da reputação corporativa e o isomorfismo mimético (SierraGarcía et al., 2013; Peters \& Romi, 2014; Alrazi et al., 2015; Fernandez-Feijoo et al., 2016).

Do ponto de vista das empresas, o capital reputacional ou efeito da "marca" das empresas Big-4é capaz de atribuir maior credibilidade à asseguração externa dos RS, quando percebido, em sua maioria, em determinado contexto social (Martínez-Ferrero \& García-Sánchez, 2018). Em mercados emergentes e desregulamentados, como asseguração externa dos RS, é possível que a incerteza sobre qual provedor é mais capacitado para realizar um trabalho de maior qualidade incentive as empresas a aderirem a práticas semelhantes e, assim, contratarem um especialista com base na sua reputação elevada em um setor em particular (FernandezFeijoo et al., 2016). Nesse ponto, as análises exploratórias demonstraram que KPMG, PWC, Deloitte e Ernst \& Young estão diferentemente distribuídas entre os setores da amostra, demonstrando que as empresas podem fazer distinção entre as Big-4 com base na percepção generalizada de seu setor.

\section{Conclusões}

A análise dos efeitos dos fatores explicativos na presença das grandes firmas de auditoria contábil no mercado de asseguração externa dos RS revelou que a prestação conjunta de auditoria contábil e a asseguração externa das informações de sustentabilidade e isomorfismo mimético 
possuem efeitos positivos, enquanto a especialização no setor e as variáveis de controle (tamanho, endividamento e rentabilidade) mostraram insignificância estatística.

Essas descobertas caracterizam a vantagem competitiva das firmas Big-4, pois esses provedores podem utilizar sua clientela de auditoria contábil como uma estratégia de inserção nesse mercado. Além disso, o capital reputacional desses profissionais permite que empresas de um mesmo setor tenham a percepção comum de que esse tipo de provedor seja o mais adequado para fornecer a asseguração externa de maior qualidade. Nas atuações individuais a KPMG foi a Big-4 de maior atuação no Brasil, destacando-se em diversos setores da amostra. As análises complementares demonstraram que as empresas Big-4 estão diferentemente distribuídas entre os setores da amostra, também revelam que a Deloitte, KPMG e PWC estão mais associadas a utilizar sua rede de clientes de auditoria contábil do que a Ernst \& Young.

Em suma, devido à livre concorrência do mercado de asseguração externa dos RS e a literatura controversa sobre qual provedor estaria mais capacitado em realizar esse trabalho, ainda que a decisão de contratar uma firma Big-4 possa estar relacionada aos efeitos da simbologia da "marca", esta pesquisa contribui para melhorar o conhecimento produzido na literatura que denotam os atributos das firmas de contabilidade Big-4 como uma ferramenta para atribuir credibilidade aos RS (O'Dwyer \& Owen, 2005; Park \& Brorson, 2005; Simnett et al., 2009; Jones \& Solomon, 2010; Gillet 2012; Fernandez-Feijoo et al., 2016). Também fornece evidências que reforçam os pilares teórico-empíricos a respeito do papel dos provedores de asseguração externa dos RS e a legitimidade das organizações, uma vez que a reputação das firmas Big-4, percebida em um contexto social mais amplo (por exemplo, setor), pode ser caracterizada como uma estratégia de legitimação empregada, principalmente, em mercados emergentes (Meyer \& Rowan, 1977; Alrazi et al., 2015).

Ainda, este estudo apresenta implicações práticas para profissionais e reguladores da área contábil, pois o reconhecimento das empresas de auditoria contábil no mercado de asseguração externa dos RS deve promover o interesse das instituições reguladoras em fornecer apoio institucional a essa prática. A prática de asseguração externa dos RS, em geral, não dispõe de padrões de auditoria específicos para a revisão de informações quanto "abrangentes" como as de sustentabilidade. A ISAE3000 (IAASB, 2013) é um padrão genérico desenvolvido para quaisquer trabalhos, diferente da auditoria das demonstrações contábeis e financeiras, e não trata especificamente das informações acerca do desempenho de sustentabilidade das organizações. Por conseguinte, o aperfeiçoamento profissional no assunto "sustentabilidade" deve ser uma preocupação para a classe contábil.

Outro ponto se refere à ausência de regulamentação dos contratos de asseguração externa dos RS, devido à forte influência das empresas relatoras sobre esses contratos. A criação de mecanismos que regulem especificamente esses contratos poderia mitigar os riscos de perda de independência do auditor.

Convém esclarecer que esta pesquisa está sujeita a algumas limitações. Com base nos resultados dessa pesquisa e na literatura pré-existente, há uma expectativa que alguns dos atributos das grandes firmas de contabilidade possam contribuir para uma maior qualidade do trabalho de asseguração externa dos RS. Entretanto, a natureza de um provedor pode não ser capaz por si só de comprovar o esse fato. Quanto a isso, este estudo não testou diretamente se as firmas Big-4 afetam a qualidade desses trabalhos, mas se os atributos que as caracterizam como tal são percebidos nesse mercado.

Ademais, o uso de um banco de dados nacional e a natureza voluntária dos processos de asseguração externa dos RS geram restrições quanto ao número de observações. Devido à baixa diversidade de subsetores e segmentos, a amostra é restrita somente a seis setores específicos. Portanto, recomenda-se para futuras pesquisas que as limitações descritas sejam de alguma forma desafiadas.

\section{Referências}

Accountability. (2005). AA 1000 Stakeholder engagement standard (Exposure Draft). London: Accountability.

Adams, C. A, \& Evans, R. (2004). Accountability, completeness, credibility and the audit expectations gap. Journal of Corporate Citizenship, 14, 97-1 15. Recuperado de https://www.jstor.org/stable/jcorpciti. 14.97.

Alrazi, B., de Villiers, C., \& van Staden, C. J. (2015). A comprehensive literature review on, and the construction of a framework for, environmental legitimacy, accountability and proactivity. Journal of Cleaner Production, 102, 44-57. doi: https://doi.org/10.1016/i.jclepro.2015.05.022.

Andon, P., Free, C., \& O'Dwyer, B. (2015). Annexing new audit spaces: challenges and adaptations. Accounting, Auditing \& Accountability Journal, 28 (8), 1400-1430. doi: https://doi.org/10.1108/AAAJ-01-2015-1932.

Bansal, P. (2005). Evolving sustainably: A longitudinal 
study of corporate sustainable development. Strategic management Journal, 26(3), 197-218. doi: https://doi. org/10.1002/smi.441

Boiral, O., \& Heras-Saizarbitoria, I. (2020). Sustainability Reporting Assurance: Creating Stakeholder Accountability Through Hyperreality?. Journal of Cleaner Production, 118596. doi: https://doi.org/10.1016/i. jclepro.2019.118596.

Boiral, O., Heras-Saizarbitoria, I., \& Brotherton, M. (2019). Assessing and improving the quality of sustainability reports: The auditors' perspective. Journal of Business Ethics. doi: https://doi.org/10.1007/s10551-017-3516. 4.

Boiral, O., Heras-Saizarbitoria, I., \& Brotherton, M. C. (2020). Professionalizing the assurance of sustainability reports: the auditors' perspective. Accounting, Auditing \& Accountability Journal, 33 (2), 309-334. doi: https://doi. org/10.1108/AAAJ-03-2019-3918.

Boiral, O., Heras-Saizarbitoria, I., Brotherton, M. C., \& Bernard, J. (2019). Ethical issues in the assurance of sustainability reports: Perspectives from assurance providers. Journal of Business Ethics, 159(4), $1111-1125$. doi: https://doi.org/10.1007/s10551-018-3840-3.

Byus, K., Deis, D., \& Reed, A. (2013). Sustainability makes auditors see green. SAM Advanced Management Journal, 78 (2), 25-63. Recuperado de https://www.proquest. $\mathrm{com} /$ docview/1427268850?pq-origsite=gscholar\&fromo penview $=$ true .

Carson, E., Fargher, N., Simon, D. T. \& Taylor, M. H. (2004). Audit fees and market segmentation-further evidence on how client size matters within the context of audit fees models. International Journal of Auditing, 8 (1), 79-91. doi: https://doi.org/10.1111/i.10991123.2004.00159.x.

Casterella, J. R., Francis, J. R., Lewis, B. L., \& Walker, P. L. (2004). Auditor industry specialization, client bargaining power, and audit pricing. Auditing: A Journal of Practice \& Theory, 23 (1), 123-140. doi: https://doi.org/10.2308/ aud.2004.23.1.123.

Chen, K.Y., Lin, K., \& Zhou, J. (2005). Audit quality and earnings management for Taiwan IPO firms. Managerial Auditing Journal, 20 (1), 86-104. doi: https://doi. org/10.1108/02686900510570722.
Cohen, J. R., \& Simnett, R. (2015). CSR and assurance services: A research agenda. Auditing: A Journal of Practice \& Theory, 34(1), 59-74. doi: https://doi. org/10.2308/ajpt-50876.

Craswell, A. T., Francis, J. R., \& Taylor, S. L. (1995). Auditor brand name reputations and industry specializations. Journal of Accounting and Economics, 20(3), 297-322. doi: https://doi.org/10.1016/0165-4101(95)00403-3.

Dando, N., \& Swift, T. (2003). Transparency and assurance: Minding the credibility gap. Journal of Business Ethics, 44, 195-200. doi:https://doi. org/10.1023/A:1023351816790.

DiMaggio, P., \& Powell, W. (1983). The iron cage revisited: Collective rationality and institutional isomorphism in organizational fields. American Sociological Review, 48 (2), 147-160.

Farooq, M. B., \& de Villiers, C. (2017). The market for sustainability assurance services: A comprehensive literature review and future avenues for research. Pacifc Accounting Review, 29 (1), 79-106. doi:https://doi. org/10.1108/PAR-10-2016-0093.

Farooq, M. B., \& de Villiers, C. (2019). Sustainability Assurance: Who Are the Assurance Providers and What Do They Do?. In: Arvidsson S. (eds) Challenges in Managing Sustainable Business. Palgrave Macmillan, Cham. doi:https://doi.org/10.1007/978-3-319-932668_6.

Fávero, L. P., \& Belfiore, P. (2017). Manual de análise de dados: estatística e modelagem multivariada com Excel $($, SPSS ${ }^{\circ}$ e Stata ${ }^{R}$. Rio de Janeiro: Elsevier.

Ferguson, A., \& Pündrich, G. (2015). Does industry specialist assurance of non-financial information matter to investors?. Auditing: A Journal of Practice \& Theory, 34(2), 121-146. doi: https://doi.org/10.2308/ajpt-50930.

Fernandez-Feijoo, B., Romero, S., \& Ruiz, S. (2016). The assurance market of sustainability reports: What do accounting firms do? Journal of Cleaner Production, 139, 1128-1137. doi: https://doi.org/10.1016/i. ¡clepro.2016.08.157.

Fonseca, A. (2010). How credible are mining corporations' sustainability reports? A critical analysis of external assurance under the requirements of the international council on mining and metals. Corporate 
Social Responsibility and Environmental Management. 17 (6), 355-370. doi: https://doi.org/10.1002/csr.230.

Gillet, C. (2012). A study of sustainability verification practices: The French case. Journal of Accounting \& Organisational Change, 8, 62-84. doi:https://doi. org/10.1108/18325911211205748.

Green, W., Taylor, S., \& Wu, J. (2017). Determinants of greenhouse gas assurance provider choice. Meditari Accountancy Research, 25(1), 114-135. doi: https://doi. org/10.1108/MEDAR-08-2016-0072.

Gürtürk A, \& Hahn, R. (2016). An empirical assessment of assurance statements in sustainability reports: smoke screens or enlightening information? Journal of Cleaner Production, 136, 30-41. doi: https://doi.org/10.1016/i. jclepro.2015.09.089.

Hodge, K., Subramaniam, N., \& Stewart, J. (2009). Assurance of sustainability reports. Impact on report users' confidence and perceptions of information credibility. Australian Accounting Review, 19(3), 178-194. doi: https://doi.org/10.1 111/j.1835-2561.2009.00056.x.

Hox, J. J. (2010). Quantitative methodology series. Multilevel analysis: Techniques and applications (2nd ed.). New York, NY, US: Routledge/Taylor \& Francis Group.

Hummel, K., Schlick, C., \& Fifka, M. (2017). The role of sustainability performance and accounting assurors in sustainability assurance engagements. Journal of Business Ethics, 154(3), 733-757. doi:https://doi.org/10.1007/ s10551-016-3410-5.

IAASB (2013). International Standard on Assurance Engagements (ISAE) 3000 Revised, Assurance Engagements Other than Audits or Reviews of Historical Financial Information. IAASB, New York. Recuperado de https://www.ifac.org/system/files/publications/files/ ISAE\%203000\%20Revised\%20-\%20for\%20IAASB.pdf.

Jones, M. J., \& Solomon, J.F. (2010). Social and environmental report assurance: some interview evidence. Accounting Forum, 34 (1), 20-31. doi:https://doi. org/10.1016/i.accfor.2009.11.002.

Kolk, A., \& Perego, P. (2010). Determinants of the adoption of sustainability assurance statements: An international investigation. Business Strategy and the Environment, 18: 182-98. doi:https://doi.org/10.1002/bse.643.
KPMG (2017). The KPMG Survey of Corporate Responsibility Reporting 2017. Zurich, Switzerland KPMG International. Recuperado de https://assets.kpmg/ content/dam $/ \mathrm{kpmg} / \mathrm{be} / \mathrm{pdf} / 2017 / \mathrm{kpmg}$-survey-ofcorporate-responsibility-reporting-2017.pdf.

Manetti, G., \& Toccafondi, S. (2012). The role of stakeholders in sustainability reporting assurance. Journal of Business Ethics, 107(3), 363-377. doi: https://doi. org/10.1007/s10551-011-1044-1.

Martínez-Ferrero, J., \& García-Sánchez, I. M. (2018). The level of sustainability assurance: The effects of brand reputation and industry specialisation of assurance providers. Journal of Business Ethics, 150 (4), 971-990. doi:https://doi.org/10.1007/s10551-016-3159-x.

Martínez-Ferrero, J., I. M. García-Sánchez \& RuizBarbadillo, E. (2018). The quality of sustainability assurance reports: The expertise and experience of assurance providers as determinants. Business Strategy and the Environment, 27(8), 1181-1 196. doi: https://doi. org/10.1002/bse.2061.

Mayhew, B. W., \& Wilkins, M. S. (2003). Audit firm industry specialization as a differentiation strategy: Evidence from fees charged to firms going public. Auditing: A Journal of Practice \& Theory, 22 (2), 33-52. doi:https:// doi.org/10.2308/aud.2003.22.2.33.

Meyer, J. W., \& Rowan, B. (1977). Institutionalized organizations: formal structure as myth and ceremony. American Journal of Sociology, 83 (2), 340-363. Recuperado de https://www.journals.uchicago.edu/doi/ abs/10.1086/226550.

O'Dwyer, B., \& Owen, D. L. (2005). Assurance statement practice in environmental, social and sustainability reporting: A critical evaluation. British Accounting Review, 37, 205-229. doi: https://doi.org/10.1016/i. bar.2005.01.005.

O'Dwyer, B., Owen, D. L., \& Unerman, J. (2011). Seeking legitimacy for new assurance norms: the case of assurance on sustainability reporting. Accounting, Organization and Society, 36 (1), 31-52. doi: https://doi.org/10.1016/i. aos. 2011.01 .002 .

Papoutsi, A., \& Sodhi, M. S. (2020). Does disclosure in sustainability reports indicate actual sustainability performance? Journal of Cleaner Production, 260, 121049. doi: https://doi.org/10.1016/i.jclepro.2020.121049. 
Park, J., \& Brorson, T. (2005). Experiences of and views on third-party assurance of corporate environmental and sustainability reports. Journal of Cleaner Production, 13, 1095-1106. doi: https://doi.org/10.1016/i. jclepro.2004.12.006.

Perego, P. (2009). Causes and consequences of choosing different assurance providers: an international study of study of sustainability reporting. International Journal of Management, 26 (3), 412-425. Recuperado de https:// repub.eur.nl/pub/19537/.

Peters, G. F., \& Romi, A. M. (2014). The association between sustainability governance characteristics and the assurance of corporate sustainability reports. Auditing: A Journal of Practice and Theory, 34 (1), 163-198. doi: https://doi.org/10.2308/ajpt-50849.

Pflugrath, G., Roebuck, P., \& Simnett, R. (2011). Impact of assurance and assurer's professional affiliation on financial analysts' assessment of credibility of corporate social responsibility information. Auditing: A Journal of Practice and Theory, 30, 239254. doi: https://doi.org/10.2308/ajpt-10047

Ruiz-Barbadillo, E., \& Martínez-Ferrero, J. (2020). Empirical analysis of the effect of the joint provision of audit and sustainability assurance services on assurance quality. Journal of Cleaner Production. doi: https://doi. org/10.1016/i.jclepro.2020.121943.
Sierra-García, L., Zorio-Grima, A., \& García-Benau, M. A. (2013). Stakeholder engagement, corporate social responsibility and integrated reporting: An exploratory study. Corporate Social Responsibility and Environmental Management, 22 (5), 286-304. doi: https://doi. org/10.1002/csr. 1345 .

Silveira, G., \& Alberton, L. (2020). Assurance dos Relatórios de Sustentabilidade no Brasil: Quais fatores podem influenciar a sua ocorrência?. Revista Universo Contábil, 15(3), 96-113. doi: http://dx.doi. org/10.4270/ruc. 2019322.

Simnett, R., Vanstraelen, A., \& Chua, W. F. (2009). Assurance on sustainability reports: An international comparison. Accounting Review, 84, 937-967. doi: https://doi.org/10.2308/accr.2009.84.3.937

Sun, W. C., Huang, H. W., Dao, M., \& Young, C. S. (2017). Auditor selection and corporate social responsibility. Journal of Business Finance \& Accounting, 44(9-10), 1241-1275. doi: https://doi.org/10.1111/ ibfa. 12260

Zhang, Z., \& Chen, H. (2019). Media coverage and impression management in corporate social responsibility reports: Evidence from China. Sustainability Accounting, Management and Policy Journal, ahead-of-print. doi: https://doi.org/10.1 108/SAMPJ-10-2018-0293. 